\title{
Complex Synchronizations in Markovian Models: a Tensor-based Proof of Product Form
}

\author{
T. H. Dao Thi \\ PRISM CNRS UMR 8144 \\ Université de Versailles St-Quentin, UniverSud \\ $45 \mathrm{Av}$. des Etats Unis, 78000 Versailles, France \\ thu-ha.dao-thi@prism.uvsq.fr
}

\author{
J.M. Fourneau \\ PRISM CNRS UMR 8144 \\ Université de Versailles St-Quentin, UniverSud \\ 45 Av. des Etats Unis, 78000 Versailles, France \\ jmf@prism.uvsq.fr
}

\begin{abstract}
We consider complex synchronizations in a generalized network of queues with signals (Gnetwork) or Stochastic Automata Networks without functions. Both models allow to describe their continuous time Markov chain as a summation of tensor (or Kronecker) products and sums of local description of queues (or automata). We give a purely algebraic proof of the product form results based on properties of the tensor products. These results generalizes many well-known results in queueing theory but also on all the models which allow a tensor based representation such as Stochastic Petri Nets or Stochastic Process Algebra.
\end{abstract}

\section{Categories and Subject Descriptors}

C.4 [Performance of Systems]: Modeling techniques; Performance attributes; G.3 [Probability and Statistics]: Markov processes

\section{Keywords}

Product form, Markovian Networks of Queues, Tensor Algebra

\section{INTRODUCTION}

In the last decade, the component based approach in performance modelling has received considerable attention. Many High Level formalisms have been developed and the translation into multidimensional Continuous Time Markov Chain (CTMC in the following) was in general proved to analyse quantitative properties of systems while qualitative ones are studied neglecting the timing information in the model (see for instance the various Stochastic Process Algebras which have been proposed). Performance analysis tools have been developed and they generally allow to perform simulations and numerical analysis.

Most of these High Level formalisms translation into DTMC are based on tensor product and sums (see a formal definition in the next section). For instance Plateau [18] has

Permission to make digital or hard copies of all or part of this work for personal or classroom use is granted without fee provided that copies are not made or distributed for profit or commercial advantage and that copies bear this notice and the full citation on the first page. To copy otherwise, to republish, to post on servers or to redistribute to lists, requires prior specific permission and/or a fee. VALUETOOLS 2009, October 20-22, Pisa, Italy

Copyright () 2009 ICST 978-963-9799-70-7

DOI 10.4108/ICST.VALUETOOLS2009.7507 proved that the transition rate matrix of a CTMC associated to a Stochastic Automata Network (SAN) is the summation of the tensor products and sums of small matrices which describe the automata:

$$
Q=\oplus_{l=1}^{n} \mathbf{L}_{\mathbf{l}}+\sum_{r=1}^{s}\left(\otimes_{i=1}^{n} \mathbf{M}_{\mathbf{i}}^{\mathbf{r}}-\mathbf{N}^{\mathbf{r}}\right),
$$

$n$ is the number of automata, $s$ is the number of synchronizations, $\mathbf{L}_{\mathbf{l}}, \mathbf{M}_{\mathbf{i}}^{\mathbf{r}}$ and $\mathbf{N}^{\mathbf{r}}$ are description matrices of the automata and $\otimes$ and $\oplus$ denote the tensor product and tensor sum. This tensor representation proved for SAN has been generalized to Stochastic Petri Nets [6] and Stochastic Process Algebra [16]. We can also describe Markovian Queueing Networks with such a tensor representation and many models in the literature are described by tensors and matrices. However this representation is only used to present the models and the analysis does not in general take advantage of the algebraic properties of these operators.

Recently, the tensor representation was also associated to analytical closed-form solutions such as product form. The main idea is the simple fact that a product form distribution is the tensor product of marginal distributions. Therefore as the solution and the transition rate matrix are both described by tensors products and sums, it may be possible to verify the balance equations using properties of tensor algebra. This approach has been used in $[10,11,8]$ to find a very simple algebraic sufficient condition for product form of steady-state solution of continuous-time or discrete-time SAN without synchronizations where interactions are limited to functional rates. This condition relies on the existence of a common vector in the kernel of all the matrices obtained when the functional rates change. The results obtained by this approach generalize Boucherie's theory of Markov chains in competition [1] and some of Robertazzi's theorems on Petri nets [19].

Furthermore, we have proved in [4] that we can generalize many results on the product form of open queueing networks using their tensor representation and algebraic arguments. In classical Markovian queueing network theory, the synchronization between the components is due to the customer movement. This is denoted as Master/Slave synchronization. The Master queue sends a customer to the Slave queue. The approach in [4] allows to prove algebraically well-known results such as Jackson network or queues with catastrophes and many recent theorems on generalized network of queues with negative customers [14] or resets [13]. Generalized networks of queues (Gnetworks for short) have been introduced in [14] and [2]. In a Gnetwork, customers can become a sig- 
nal when they move from one queue to another one. The signals visit queue, they do not require service but they have an immediate action upon the queue. A signal may disappear if some conditions are fulfilled at the queue it is visiting or it may continue its visit to another queue. Therefore Gnetwork with signals are associated to much more complex synchronizations between queues. In [9] we have shown that the same algebraic techniques we have developed for simple synchronization such as Master/Slave also allows to prove that networks with a complex synchronization denoted as a Domino has a product form steady-state distribution. The domino synchronization is described in section 5 .

Here we consider a much more complex set of synchronizations between an arbitrary number of automaton (or equivalently a signal propagating among an arbitrary number of queues with an arbitrary effect on each of them). The propagation of the signal may depend on the state of the queue it is visiting. Using the same kind of algebraic arguments we prove a sufficient condition for the Markovian model to have a product form steady-state distribution. We also show that this result generalizes many known results in queueing theory. We present the models using Stochastic Automata Network of Markovian networks of queues with signals. Both formalisms are equivalent. The real property we use is the representation of the transition rate matrix using tensor product (i.e. refer to Eq. 1).

The rest of the paper is as follows. First in section 2 we give a brief description of tensor (or Kronecker) operators on matrices and we give a first result on networks with jump over blocking to introduce the technique and show that algebraic techniques provide a more general sufficient condition than the one which were previously known. Then in section 3 we present the notation and concepts about synchronizations. A synchronization is defined as an ordered list of automaton (or queue visited by the signal). Section 4 is devoted to the main theorem on the synchronizations with product form. We give two proofs of the result. The first one (the simplest) assume that there is no duplication in the list while the second proof does not require such an assumption. This proof is postponed into an appendix for the sake of readability. Finally in section 5 we present many examples to illustrate the approach.

\section{KRONECKER ALGEBRA AND PRODUCT FORM}

In this paper, we restrict ourself to continuous-time SAN without functions. The generator is based on the tensor sum and product of local components. Recall that with

$$
A=\left(\begin{array}{ll}
a_{11} & a_{12} \\
a_{21} & a_{22}
\end{array}\right) \quad \text { and } B=\left(\begin{array}{lll}
b_{11} & b_{12} & b_{13} \\
b_{21} & b_{22} & b_{23} \\
b_{31} & b_{32} & b_{33}
\end{array}\right) \text {, }
$$

the tensor product $A \otimes B$ is:

$$
\left(\begin{array}{ccc|ccc}
a_{11} b_{11} & a_{11} b_{12} & a_{11} b_{13} & a_{12} b_{11} & a_{12} b_{12} & a_{12} b_{13} \\
a_{11} b_{21} & a_{11} b_{22} & a_{11} b_{23} & a_{12} b_{21} & a_{12} b_{22} & a_{12} b_{23} \\
a_{11} b_{31} & a_{11} b_{32} & a_{11} b_{33} & a_{12} b_{31} & a_{12} b_{32} & a_{12} b_{33} \\
\hline a_{21} b_{11} & a_{21} b_{12} & a_{21} b_{13} & a_{22} b_{11} & a_{22} b_{12} & a_{22} b_{13} \\
a_{21} b_{21} & a_{21} b_{22} & a_{21} b_{23} & a_{22} b_{21} & a_{22} b_{22} & a_{22} b_{23} \\
a_{21} b_{31} & a_{21} b_{32} & a_{21} b_{33} & a_{22} b_{31} & a_{22} b_{32} & a_{22} b_{33}
\end{array}\right),
$$

and the tensor sum $A \oplus B$ is given by: $A \otimes I_{B}+I_{A} \otimes B$ where $I_{A}$ and $I_{B}$ are Identity matrices whose size is respectively equal to the size of $A$ and $B$. The tensor product and sums have many algebraic properties (see [7] or [17] for proofs). We only give some of them in the following for the sake of completeness as they are used in the proofs. Note that these properties have also been used in $[10,11,8,4]$ to prove product form steady-state distributions for other types of SAN. The key property is the fact that a product form solution of $n$ distributions $\left(\pi_{l}\right)_{l=1 . . n}$ is a tensor product: i.e. it is equal to $C \pi_{1} \otimes \pi_{2} \otimes \ldots \otimes \pi_{n}$ where $C$ is a normalization constant.

Property 2.1 (Basic Properties of Tensor). Let $A, B, C$, and $D$ be arbitrary matrices, the following properties hold:

- Associativity: $(A \otimes B) \otimes C=A \otimes(B \otimes C)$.

- Distributivity over Addition:

$$
(A+C) \otimes(B+D)=A \otimes B+A \otimes D+C \otimes B+C \otimes D .
$$

- Compatibility with matrix multiplication: For all vectors $\pi_{A}$ and $\pi_{B}$ whose sizes are consistent we have:

$$
\left(\pi_{A} \otimes \pi_{B}\right)(A \otimes B)=\left(\pi_{A} A\right) \otimes\left(\pi_{B} B\right) .
$$

We assume that the state space is the Cartesian product of the states of the components (i.e. all the states are reachable). The reachable state space is in general only a subset of this Cartesian product.

Let us now give some notation and definitions on the matrices used to design the example of models.

$I d_{n}$ the identity matrix of size $n$,

$U p_{n}$ the matrix of size $n$ full of 0 except the main upper diagonal which is 1 , this matrix is used to represent arrival of customers,

$L_{o w}$ the matrix of size $n$ full of 0 except the main lower diagonal which is 1 ,this matrix is used to represent departure of customers,

$I_{n}$ size $n$, all elements equal to 0 except $I_{n}(n, n)=1$,

$I_{n}^{0}$ size $n$, all elements equal to 0 except $I_{n}^{0}(1,1)=1$.

Note that we accept the case where $n=\infty$. In that case, the index $n$ will be omitted.

Definition 2.2. Let $M$ be a square matrix. The matrix $\sigma(M)$ is a diagonal matrix with the size of $M$ such that for all index $i, \sigma(M)[i, i]=\sum_{j} M[i, j]$.

Let us now prove that the basic properties of tensor are sufficient to prove that the steady-state solution of a model described by Kronecker products has product form.

\subsection{Three queues with jump over blocking}

Before giving a general and complicated definition of the synchronization with multiple steps in section 3 , let us begin with a simpler example to illustrate: the jump over blocking queue.

Consider a network consisting of 3 queues: $Q 1, Q 2$, and $Q 3$. Queue $Q 2$ has a finite buffer with size $K$. Arrivals to queue $Q 1$ follow independent Poisson processes. The other queues do not receive customers from the the outside. Services have exponential durations and they are independent. Customers leaving $Q 3$ depart from the network. The arrival 
rate from the outside to queue $Q 1$ is $\lambda_{1}$. The service rate at queue $i$ is $\mu_{i}$. At the completion of its service in queue $Q 2$, a customer jumps to queue $Q 3$. When its service at queue $Q 1$ is completed, a customer jumps to queue $Q 2$ if there is a place available in the buffer; otherwise it jumps to queue $Q 3$. Such a network is known to have a product form solution [2, $5]$.

To give a completely algebraic proof, we do the following. We do not take into account the exact representation of an arrival or a service but we describe them as transition rate matrices. More precisely, arrivals in queue $Q 1$ and service in $Q 3$ are local transitions and they are respectively represented by transition rate matrices $L_{1}$ and $L_{3}$. A customer movement between queue $Q 2$ and queue $Q 3$ is described by matrix $D_{2}$ for queue $Q 2$ and transition probability matrix $S_{1}$ for $Q 3 . D_{2}$ is a transition rate matrix without diagonalisation (i.e. $\sigma\left(D_{2}\right) \neq 0$ ). When a customer succeeds to move from $Q_{1}$ to $Q_{2}$, its behaviour is modelled by matrix $D_{1}$ (with $\sigma\left(D_{1}\right) \neq 0$ ) for queue $Q 1$ and transition probability matrix $S_{2}$ for queue $Q 2$. When the movement is refused by queue $Q 2$ and the customer moves from $Q 1$ to $Q 3$, the model is represented by $D_{1}$ on queue $Q 1, S_{4}$ for $Q 2$ and $S_{3}$ for queue Q3. Thus the transition rate matrix of the CTMC is:

$$
\begin{aligned}
Q & =L_{1} \oplus L_{3} \\
& +I d \otimes D_{2} \otimes S_{1}-I d \otimes \sigma\left(D_{2}\right) \otimes \sigma\left(S_{1}\right) \\
& +D_{1} \otimes S_{2} \otimes I d-\sigma\left(D_{1}\right) \otimes \sigma\left(S_{2}\right) \otimes I d \\
& +D_{1} \otimes S_{4} \otimes S_{3}-\sigma\left(D_{1}\right) \otimes \sigma\left(S_{4}\right) \otimes \sigma\left(S_{3}\right)
\end{aligned}
$$

In the "language" of synchronization, in this example, a synchronization $r$ may have 2 or 3 steps. At each step, an automaton will be effected by a matrix (an automaton does not appear in the synchronization associated to the Identity matrix). Let us give the detail in the following tabular

\begin{tabular}{|c|c|c|c|}
\hline$r=(Q 2, Q 3)$ & & $\mathrm{Q} 2$ & $\mathrm{Q} 3$ \\
$M$ & & $D_{2}$ & $S_{1}$ \\
\hline$r=(Q 1, Q 2)$ & $\mathrm{Q} 1$ & $\mathrm{Q} 2$ & \\
$M$ & $D_{1}$ & $S_{2}$ & \\
\hline$r=(Q 1, Q 2, Q 3)$ & $\mathrm{Q} 1$ & $\mathrm{Q} 2$ & $\mathrm{Q} 3$ \\
$M$ & $D_{1}$ & $S_{4}$ & $S_{3}$ \\
\hline
\end{tabular}

To illustrate, the explicit representing of the matrices will be given after the algebraic proof.

TheOREM 2.3. Assume that $Q$ is ergodic and that we have $S_{4}=\sigma\left(S_{4}\right)$ and $\sigma\left(S_{2}\right)+\sigma\left(S_{4}\right)=I$. Let $X_{i}$ the size of queue indexed by $i$. Assume that the following conditions hold:

$$
\left\{\begin{array}{l}
g_{1} D_{1}=\Gamma^{12} g_{1} \\
g_{2}\left(D_{2}+\Gamma^{12} S_{4}\right)=\Gamma^{23} g_{2} \\
g_{1} H_{1}=0, \quad g_{2} H_{2}=0, \quad g_{3} H_{3}=0 \\
g_{3} S_{1}=g_{3} S_{3}
\end{array}\right.
$$

where

$$
\left\{\begin{array}{l}
H_{1}=L_{1}+D_{1}-\sigma\left(D_{1}\right) \\
H_{2}=D_{2}-\sigma\left(D_{2}\right)+\Gamma^{12}\left(S_{2}-\sigma\left(S_{2}\right)\right), \\
H_{3}=L_{3}+\Gamma^{23}\left(S_{1}-\sigma\left(S_{1}\right)\right),
\end{array}\right.
$$

then the steady-state solution has product form:

$$
\operatorname{Pr}\left(X_{1}, X_{2}, X_{3}\right)=C \prod_{i=1}^{3} g_{i}\left(X_{i}\right)
$$

We just give here the proof to highlight the tensor properties we need. Assume that the steady-state solution has product form and consider $\operatorname{Pr}\left(X_{1}, X_{2}, X_{3}\right) Q$. We will verify that the product is equal to zero. Because of the distributivity and the compatibility with ordinary product we have:

$$
\begin{aligned}
\left(g_{1} \otimes g_{2} \otimes g_{3}\right) Q & =g_{1} L_{1} \otimes g_{2} \otimes g_{3} \\
& +g_{1} \otimes g_{2} \otimes g_{3} L_{3} \\
& +g_{1} \otimes g_{2} D_{2} \otimes g_{3} S_{1} \\
& -g_{1} \otimes g_{2} \sigma\left(D_{2}\right) \otimes g_{3} \sigma\left(S_{1}\right) \\
& +g_{1} D_{1} \otimes g_{2} S_{2} \otimes g_{3} \\
& -g_{1} \sigma\left(D_{1}\right) \otimes g_{2} \sigma\left(S_{2}\right) \otimes g_{3} \\
& +g_{1} D_{1} \otimes g_{2} S_{4} \otimes g_{3} S_{3} \\
& -g_{1} \sigma\left(D_{1}\right) \otimes g_{2} \sigma\left(S_{4}\right) \otimes g_{3} \sigma\left(S_{3}\right) .
\end{aligned}
$$

Remember that $g_{1} D_{1}=\Gamma^{12} g_{1}$ and that $g_{3} S_{1}=g_{3} S_{3}$. Note that $\sigma\left(S_{1}\right)=\sigma\left(S_{3}\right)=I$. Substitute into the equation to obtain:

$$
\begin{aligned}
\left(g_{1} \otimes g_{2} \otimes g_{3}\right) Q & =g_{1} L_{1} \otimes g_{2} \otimes g_{3} \\
& +g_{1} \otimes g_{2} \otimes g_{3} L_{3} \\
& +g_{1} \otimes g_{2} D_{2} \otimes g_{3} S_{1} \\
& -g_{1} \otimes g_{2} \sigma\left(D_{2}\right) \otimes g_{3} \\
& +g_{1} \otimes g_{2} \Gamma^{12} S_{2} \otimes g_{3} \\
& -g_{1} \sigma\left(D_{1}\right) \otimes g_{2} \sigma\left(S_{2}\right) \otimes g_{3} \\
& +g_{1} \otimes g_{2} \Gamma^{12} S_{4} \otimes g_{3} S_{1} \\
& -g_{1} \sigma\left(D_{1}\right) \otimes g_{2} \sigma\left(S_{4}\right) \otimes g_{3} .
\end{aligned}
$$

Factorize the 6 th with the 8 th terms of right hand side, do the same for the $3 \mathrm{rd}$ and the 7 th terms. Remember that $\sigma\left(S_{2}\right)+\sigma\left(S_{4}\right)=I$ to get:

$$
\begin{aligned}
\left(g_{1} \otimes g_{2} \otimes g_{3}\right) Q & =g_{1} L_{1} \otimes g_{2} \otimes g_{3} \\
& +g_{1} \otimes g_{2} \otimes g_{3} L_{3} \\
& +g_{1} \otimes g_{2}\left(D_{2}+\Gamma^{12} S_{4}\right) \otimes g_{3} S_{1} \\
& -g_{1} \otimes g_{2} \sigma\left(D_{2}\right) \otimes g_{3} \\
& +g_{1} \otimes g_{2} \Gamma^{12} S_{2} \otimes g_{3} \\
& -g_{1} \sigma\left(D_{1}\right) \otimes g_{2} \otimes g_{3}
\end{aligned}
$$

Now we take into account that $g_{2}\left(D_{2}+\Gamma^{12} S_{4}\right)=g_{2} \Gamma^{23}$. We substitute in the equation and we factorize the second and the third terms, and the first and the last terms:

$$
\begin{aligned}
\left(g_{1} \otimes g_{2} \otimes g_{3}\right) Q & =g_{1}\left(L_{1}-\sigma\left(D_{1}\right)\right) \otimes g_{2} \otimes g_{3} \\
& +g_{1} \otimes g_{2} \otimes g_{3}\left(L_{3}+\Gamma^{23} S_{1}\right) \\
& -g_{1} \otimes g_{2} \sigma\left(D_{2}\right) \otimes g_{3} \\
& +g_{1} \otimes g_{2} \Gamma^{12} S_{2} \otimes g_{3}
\end{aligned}
$$

We add and we subtract $g_{1} D_{1} \otimes g_{2} \otimes g_{3}$ and we note that $g_{1} D_{1} \otimes g_{2}=g_{1} \Gamma^{12} \otimes g_{2}=g_{1} \otimes g_{2} \Gamma^{12}$. Similarly we add and we subtract $g_{1} \otimes g_{2}\left(D_{2}+\Gamma^{12} S_{4}\right) \otimes g_{3}$ and we take into account that $g_{2}\left(D_{2}+\Gamma^{12} S_{4}\right) \otimes g_{3}=g_{2} \otimes \Gamma^{23} g_{3}$. After substitution and factorization we get:

$$
\begin{aligned}
\left(g_{1} \otimes g_{2} \otimes g_{3}\right) Q & =g_{1}\left(L_{1}+D_{1}-\sigma\left(D_{1}\right)\right) \otimes g_{2} \otimes g_{3} \\
& +g_{1} \otimes g_{2} \otimes g_{3}\left(L_{3}+\Gamma^{23}\left(S_{1}-I\right)\right) \\
& +g_{1} \otimes g_{2} G \otimes g_{3}
\end{aligned}
$$

where $G=\left(D_{2}-\sigma\left(D_{2}\right)+\Gamma^{12}\left(S_{2}+S_{4}-I\right)\right.$. Finally some terms cancel because $I=\sigma\left(S_{2}\right)+\sigma\left(S_{4}\right)$ and $S_{4}=\sigma\left(S_{4}\right)$. We recognize matrices $M_{1}, M_{2}$ and $M_{3}$. Thus,

$$
\begin{aligned}
\left(g_{1} \otimes g_{2} \otimes g_{3}\right) Q & =g_{1} H_{1} \otimes g_{2} \otimes g_{3} \\
& +g_{1} \otimes g_{2} \otimes g_{3} H_{3} \\
& +g_{1} \otimes g_{2} H_{2} \otimes g_{3}
\end{aligned}
$$

Therefore $\left(g_{1} \otimes g_{2} \otimes g_{3}\right) Q=0$. The solution has product form.

Let us discuss about the above assumptions. Matrices $H_{1}$, $H_{2}$ and $H_{3}$ may be seen as the total transition rate matrix 
associated to $Q 1, Q 2$ and $Q 3$ in isolation. These generators take into account the local transitions (matrices $L_{i}$ ), the emission of signals (matrices $D_{i}$ ) and the effect of signals (matrices $S_{i}$ ). The eigenvalues $\Gamma^{12}$ and $\Gamma^{23}$ can be consider as the total arrival rate to queues $Q 2$ and $Q 3$

Now let us turn back to the network of queue and give the representation of the matrices used to describe the ordinary queueing model:

$$
\begin{array}{r}
L_{1}=\lambda_{1} U p-\sigma\left(\lambda_{1} U p\right), L_{2}=0, L_{3}=\mu_{3} \text { Low }-\sigma\left(\mu_{3} \text { Low }\right), \\
D_{1}=\mu_{1} L o w, \quad D_{2}=\mu_{2} L_{o w}, \quad S_{1}=U p, \\
S_{2}=U p_{K}, \quad S_{3}=U p, \quad S_{4}=I_{K} .
\end{array}
$$

Clearly the assumptions of the theorem are satisfied. Solving the system, one obtains that $g_{i}$ has geometric distribution with rate $\rho_{i}$ given by

$$
\rho_{1}=\frac{\lambda_{1}}{\mu_{1}}, \quad \rho_{2}=\frac{\Gamma^{12}}{\mu_{2}}, \quad \rho_{3}=\frac{\Gamma^{23}}{\mu_{3}},
$$

where $\Gamma^{12}=\mu_{1} \rho_{1}\left(=\lambda_{1}\right)$ and $\Gamma^{23}=\mu_{2} \rho_{2}\left(=\lambda_{1}\right)$.

Note however that the theorem and the proof is more general than the well known result of queues with jump over blocking. We only require that $g_{3} S_{1}=g_{3} S_{3}$ while the classical results require that the effect of a customer who jumps over $Q 2$ is identical to the effect of a customer completing its service at $Q 2$. One can restate this last condition as $S_{1}=S_{3}$. Clearly the algebraic proof can deal with more abstracted behaviour of customers and signals. It also allows to obtain weaker sufficient conditions.

\section{MULTISTEP SYNCHRONIZATION}

We now consider synchronizations with multiple steps. A synchronization takes place according to the order of a list of automata or queues. For instance we can consider networks with finite buffers or networks with signals which trigger customers.

The generator is given by:

$$
Q=\oplus_{i=1}^{N} F_{i}+\sum_{r \in \mathcal{S}} \otimes_{i=1}^{N} M_{i}^{r}
$$

where $\mathcal{S}$ is a set of all synchronizations. $F_{i}$ denotes the local transition in automaton $i$ and does not contain normalization. Here we slightly change the description of the model to simplify the proofs. We have $L_{i}=F_{i}-\sigma\left(F_{i}\right)$. Without loss of generality we now do not suppose that matrices $F_{i}$ and $Q$ have the sum of each line equal to 0 (we do not take into account the normalization term in the matrix). To prove that the Markov chain modelling the queueing network or the SAN has a steady-state distribution $\pi$, it is equivalent to have $\pi Q=\pi \sigma(Q)$.

Let us now detail all the possible synchronizations. Synchronization $r$ consists a list of pairs of an automaton and a type:

$$
\operatorname{List}(r)=\left\{\left(l_{1}, t_{1}\right),\left(l_{2}, t_{2}\right), \cdots,\left(l_{n(r)}, t_{n(r)}\right)\right\},
$$

where $l_{i}$ is an automaton and $t_{i}$ is a type in set $\mathcal{T}$ and suppose that $|\operatorname{List}(r)| \geq 2$. Here, for a multistep synchronization $r$, there are $n(r)$ consecutive steps (or $n(r)$ automatons (queues) will be effected). At step $i$, the synchronization $r$ is at automaton $l_{i}$ and the "action" type to $l_{i}$ is $t_{i}$ (in the example at the end of this section, $t_{i}$ can be an "arrival" or a "departure" or a "signal"). For synchronization $r$ associated to a list given in (3), let us define:

$$
\operatorname{List}^{1}(r)=\left\{l_{1}, l_{2}, \cdots, l_{n}\right\} .
$$

Furthermore, define $\operatorname{Order}(i, r)$ the sequence in order of automaton $i$ 's appearance in List $(r)\left(\operatorname{List}^{1}(r)\right)$, or

$$
\operatorname{Order}(i, r)=\left\{\alpha_{1}, \alpha_{2}, \cdots, \alpha_{n} \mid i_{j}<i_{j+1}\right\}
$$

satisfies $\operatorname{List}^{1}(r)(k)=(i)$ iff $k \in \operatorname{Order}(i, r)$.

Matrices $M_{i}^{r}$ are determined as follows:

1. If $\operatorname{Order}(i, r)=\emptyset\left(i \notin \operatorname{List}^{1}(r)\right.$, or the automaton $i$ does not appear in the synchronization $r$ )then $M_{i}^{r}=$ Id.

2. If $\operatorname{Order}(i, r)=\left\{\alpha_{1}, \cdots, \alpha_{n}\right\}$ (the automaton $i$ appears $n$ times in the synchronization $r$ ) then

$$
M_{i}^{r}=M_{i}^{r}(1) M_{i}^{r}(2) \cdots M_{i}^{r}(n),
$$

where $M_{i}^{r}(k)$ is defined by:

$$
\begin{cases}E^{\left(l_{\alpha_{k}}, t_{\alpha_{k}}\right),\left(l_{\alpha_{k}+1}, t_{\alpha_{k}+1}\right)}, & 1<k<n ; \\ E^{\left(l_{\alpha_{1}}, t_{\alpha_{1}}\right),\left(l_{\alpha_{1}+1}, t_{\alpha_{1}+1}\right)}, & k=1, i \neq l_{1} ; \\ E^{\left(l_{\alpha_{n}}, t_{\alpha_{n}}\right),\left(l_{\alpha_{n}+1}, t_{\alpha_{k}+1}\right)}, & k=n, i \neq l_{n(r)} ; \\ E_{0}^{\left(l_{n(r)}, t_{n(r)}\right)}, & k=n, i=l_{n(r)} ; \\ E_{0}^{\left(l_{1}, t_{1}\right),\left(l_{2}, t_{2}\right)}, & k=1, i=l_{1} ;\end{cases}
$$

All matrices $E$ are positive. Figure 1 illustrates a synchronization with $n(r)$ steps and the corresponding matrices.

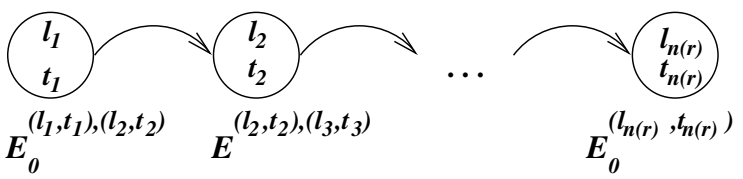

Figure 1: A synchronization $r$ with $n(r)$ steps

Let us define:

$$
\text { First }=\left\{t \in \mathcal{T} \mid E_{0}^{(j, t)}=E^{(j, t),(i, u)}=0, \forall j, i, u\right\}
$$

the set of all types which can be present only at the first step of a synchronization. Assume that the set $\mathcal{T} \backslash$ First can be decomposed in $T$ disjoint subsets

$$
\mathcal{T} \backslash \text { First }=\cup_{a=1}^{T} \mathcal{T}^{a}
$$

which satisfies for all $v \in \mathcal{T}^{a}$ :

$$
E^{(i, u),(j, v)}=E^{(i, u),\left(j, \mathcal{T}^{a}\right)}, \quad E_{0}^{(i, u),(j, v)}=E_{0}^{(i, u),\left(j, \mathcal{T}^{a}\right)} .
$$

Equation (6) states that the step before a step of all type in $\mathcal{T}^{a}$ have the same matrix. In general, $\left|\mathcal{T}^{a}\right|=1$. However, in some special cases, for example the jump over blocking network previously studied, $\left|\mathcal{T}^{a}\right|>1$.

Let us define now $E^{(j, v)}=E_{0}^{(j, v)}+\sum_{k, a} E^{(j, v),\left(k, \mathcal{T}^{a}\right)}$. This is the "total action" applied on automaton $j$ with type $v$ : it is the sum of a synchronization's last step and all intermediate steps from automaton $j$ with type $v$ to another automaton $k$ with a type in $\mathcal{T}^{a}$. Define also

$$
E^{\left(j, \mathcal{T}^{a}\right)}=\sum_{v \in \mathcal{T}^{a}} E^{(j, v)}
$$


the "total action" upon automaton $j$ with type in $\mathcal{T}^{a}$. Suppose that

$$
\sigma\left(E^{\left(j, \mathcal{T}^{a}\right)}\right)=I d
$$

Define

$$
\begin{aligned}
& E^{(i, u), j}=\sum_{v} \mathbb{1}_{E^{(i, u),(j, v)} \neq 0} E^{(j, v)}, \\
& E_{0}^{(i, u), j}=\sum_{v} \mathbb{1}_{E_{0}^{(i, u),(j, v)} \neq 0} E^{(j, v)},
\end{aligned}
$$

the "total action" upon automaton $j$ from automaton $i$ with type $u$ at an intermediate step and at a first step.

Assume that the following conditions hold:

$$
\begin{aligned}
& \sigma\left(E^{(i, u), j}\right)\left(\sigma\left(E^{(i, u), j}\right)-I d\right)=0, \\
& \sigma\left(E_{0}^{(i, u), j}\right)\left(\sigma\left(E_{0}^{(i, u), j}\right)-I d\right)=0 .
\end{aligned}
$$

Conditions (8) mean that at one step (say $(i, u)$ ), the summation of the matrices for all the possible synchronizations after this step to another automaton (say $j$ ) is a stochastic matrix $\left(\sigma\left(E^{(i, u), j}\right)=I d, \sigma\left(E_{0}^{(i, u), j}\right)=I d\right)$. It implies that

$$
\sigma\left(M E^{(i, u), j}\right)=\sigma(M), \quad \sigma\left(M E_{0}^{(i, u), j}\right)=\sigma(M),
$$

for all matrix $M$ with convenient size. This is the generalization of condition $\left(\sigma\left(S_{4}\right)+\sigma\left(S_{2}\right)=I\right)$ in section 2 .

The hypothesis (7) is interpreted as: if $\sigma\left(E^{(i, u), j}\right)=I d$ (or $\sigma\left(E_{0}^{(i, u), j}\right)=I d$ ) then $E^{(i, u), j}$ (or $E_{0}^{(i, u), j}$ ) is the sum taken over all $v$ in some subset $\mathcal{T}^{a}$.

For a set $\mathcal{T}^{a}$ and a type $v$ in $\mathcal{T}^{a}$, define

$$
\operatorname{Prev}(v)=\left\{u \in \mathcal{T} \mid \exists i, j \text { s.t } E^{(i, u),(j, v)}+E_{0}^{(i, u),(j, v)} \neq 0\right\}
$$

the set of all types can be presented before the type $v$ in some synchronization.

To illustrate these concepts, let us first consider some examples.

EXAMPLE 3.1. Three automata studied in section 2.1.

Set $\mathcal{T}$ is $\mathcal{T}=\{d, a, f\}$, where "d", "a" and "f" stand for "departure", "arrival" and "full". Set First is given by First $=$ $\{d\}$, and $T=1, \mathcal{T}^{1}=\{a, f\}$.

Set Prev is $\operatorname{Prev}(a)=\{d, f\}, \quad \operatorname{Prev}(f)=\{d\}$.

The matrices which define the synchronizations are given as in section 2.1. The condition (7) (and (8)) are satisfied, as $\sigma\left(U p_{K}+I_{K}\right)=I d$.

EXAMPLE 3.2. We now consider the general jump over blocking network with more than three queues. As in the previous case, sets $\mathcal{T}$, First and $\mathcal{T}^{a}$ are given by

$$
\mathcal{T}=\{d, a, f\}, \quad \text { First }=\{d\}, \quad \mathcal{T}^{1}=\{a, f\} .
$$

However, in general, set Prev is

$$
\operatorname{Prev}(a)=\operatorname{Prev}(f)=\{d, f\} .
$$

The matrices used to define the synchronizations will be given later in the section 5.1.

EXAMPle 3.3. A signal triggers customer departure as in a Gnetwork with triggers. Set $\mathcal{T}$ is

$$
\mathcal{T}=\{d, s, a\}
$$

where "d", "a" and "s" stand for "departure", "arrival" and "signal". Set First is given by First $=\{d\}$, and $T=2, \mathcal{T}^{1}=$ $\{a\}, \mathcal{T}^{2}=\{s\}$.

Set Prev is given by $\operatorname{Prev}(a)=\operatorname{Prev}(s)=\{d, s\}$. For the definition of the matrices associated with synchronizations, see section 5.3 .

\section{PRODUCT FORM SOLUTION}

\subsection{Main theorem}

We now state the main result of this paper.

TheOREM 4.1. Consider a SAN with $N$ automata or a Gnetwork with signals. Assume that the CTMC obtained from this high level specification is ergodic. Let $\mathcal{S}$ be the set of synchronizations with multiple steps. Assume that $g_{i}$ is an eigenvector of all matrices $\left(E_{P r e v\left(\mathcal{T}^{a}\right), \mathcal{T} a}^{i j}\right)_{a \leq T}$, where

$$
\begin{aligned}
E_{\operatorname{Prev}\left(\mathcal{T}^{a}\right) \mathcal{T}^{a}}^{i j}= & \sum_{u \in \operatorname{Prev}\left(\mathcal{T}^{a}\right)}\left\{E_{0}^{(i, u),\left(j, \mathcal{T}^{a}\right)}\right. \\
& \left.+\sum_{\substack{k \neq i \\
u \in \mathcal{T}^{b}}} \Gamma_{\operatorname{Prev}\left(\mathcal{T}^{b}\right) \mathcal{T}^{b}}^{k i} E^{(i, u),\left(j, \mathcal{T}^{a}\right)}\right\},
\end{aligned}
$$

where $\Gamma_{P r e v\left(\mathcal{T}^{b}\right) \mathcal{T}^{b}}^{k i}$ is the eigenvalue of the matrix $E_{\operatorname{Prev}\left(\mathcal{T}^{b}\right) \mathcal{T}^{b}}^{k i}$.

If $g_{i}$ is in the kernel of matrix

$$
\begin{aligned}
H_{i}= & F_{i}-\sigma\left(F_{i}\right) \\
& +\sum_{\substack{j \neq i \\
a \leq T}}\left(E_{\operatorname{Prev}\left(\mathcal{T}^{a}\right) \mathcal{T}^{a}}^{i j}-\sigma\left(E_{\operatorname{Prev}\left(\mathcal{T}^{a}\right) \mathcal{T}^{a}}^{i j}\right)\right) \\
& +\sum_{\substack{j \neq i \\
a \leq T}} \Gamma_{\operatorname{Prev}\left(\mathcal{T}^{a}\right) \mathcal{T}^{a}}^{j i}\left(E_{0}^{\left(i, \mathcal{T}^{a}\right)}-\sigma\left(E_{0}^{\left(i, \mathcal{T}^{a}\right)}\right)\right),
\end{aligned}
$$

where $E_{0}^{\left(i, \mathcal{T}^{a}\right)}=\sum_{u \in \mathcal{T}^{a}}\left(E_{0}^{(i, u)}\right)$, then the steady-state distribution has product form solution:

$$
\operatorname{Pr}\left(X_{1}, X_{2}, \cdots, X^{N}\right)=C \prod_{i=1}^{N} g_{i}\left(X_{i}\right),
$$

and $C$ is a normalization constant.

In (11), matrix $E_{\operatorname{Prev}\left(\mathcal{T}^{a}\right) \mathcal{T}^{a}}^{i j}$ can be viewed as the "total action matrix" applied to automaton $j$ with a type in $\mathcal{T}^{a}$ from automaton $i$. The value $\Gamma_{\operatorname{Prev}\left(\mathcal{T}^{a}\right) \mathcal{T}^{a}}^{k i}$ can be consider as the "total arrival rate" to automaton $j$ with a type in $\mathcal{T}^{a}$ from automaton $i$.

\subsection{A simple case}

The complete proof of theorem (4.1) will be given in Appendix. In this section, we consider a simpler case: each automaton can appear at most once in List $^{1}$ of one synchronization and set $\mathcal{T}^{a}$ has only one element $\left|\mathcal{T}^{a}\right|=1$. In this case, one has

$$
|\operatorname{Order}(i, r)| \leq 1 \text {, }
$$

hence, the form of $M_{i}^{r}$ given in (4) is the product of at most one factor.

For simplicity, from now on, all the written tensor product will be automatically rewritten in the natural order. It simply means that $g_{i_{1}} P_{i_{1}} \otimes \cdots \otimes g_{i_{N}} P_{i_{N}}$ is the product $g_{1} P_{1} \otimes \cdots \otimes g_{N} P_{N}$. 
Now, we will prove Theorem 4.1 in this simplified case. As mentioned before, we will prove that $\pi Q=\pi \sigma(Q)$. Define

$$
\mathcal{S}(n)=\{r \in \mathcal{S}|| \operatorname{List}(r) \mid=n\}
$$

the set of all synchronizations with length $n$. In the following definitions, for synchronization $r$ in $\mathcal{S}(n)$,

$$
\operatorname{List}(r)=\left\{\left(l_{1}, t_{1}\right), \cdots,\left(l_{n}, t_{n}\right)\right\} .
$$

Let us define $Q(n)$ by:

$$
Q(n)=\sum_{r \in \mathcal{S}(n)} M_{1}^{r} \otimes \cdots \otimes M_{N}^{r} .
$$

In this case, $Q(n)$ is the sum over all $\mathcal{S}(n)$ of the tensor product $E_{0}^{\left(l_{1}, t_{1}\right),\left(l_{2}, t_{2}\right)} \otimes E^{\left(l_{2}, t_{2}\right),\left(l_{3}, t_{3}\right)} \otimes \cdots \otimes E_{0}^{\left(l_{n}, t_{n}\right)}$ in a convenient order. Furthermore we define $\widehat{Q}(n)$ and $\widetilde{Q}(n)$ by:

$$
\begin{aligned}
& \widehat{Q}(n)=\sum_{r \in \mathcal{S}(n)} M_{1}^{r} \otimes \cdots \otimes \widehat{M}_{l_{1}}^{r} \cdots \otimes M_{N}^{r}, \\
& \widetilde{Q}(n)=\sum_{r \in \mathcal{S}(n)} M_{1}^{r} \otimes \cdots \otimes \widetilde{M}_{l_{n}}^{r} \cdots \otimes M_{N}^{r},
\end{aligned}
$$

where

$$
\begin{aligned}
\widehat{M}_{l_{1}}^{r} & =\sum_{k \notin \operatorname{List}^{1}(r)} \Gamma_{\operatorname{Prev}\left(t_{1}\right)\left\{t_{1}\right\}}^{k l_{1}} E^{\left(l_{1}, t_{1}\right),\left(l_{2}, t_{2}\right)}, \\
\widetilde{M}_{l_{n}}^{r} & =\sum_{k \notin \operatorname{List}^{1}(r)} \sum_{t \in \mathcal{T} \backslash \text { First }} E^{\left(l_{n}, t_{n}\right),(k, t)} .
\end{aligned}
$$

For the sake of simplification, for $\mathcal{T}^{a}=\{u\}$, let us denote by $E_{u}^{i j}$ and $\Gamma_{u}^{i j}$ the terms $E_{\operatorname{Prev}\left(\mathcal{T}^{a}\right) \mathcal{T}^{a}}^{i j}$ and $\Gamma_{\operatorname{Prev}\left(\mathcal{T}^{a}\right) \mathcal{T}^{a}}^{i j}$, respectively. Equations $g_{i} E_{u}^{i j}=\Gamma_{u}^{i j} g_{i}$ and (11) imply:

$$
\begin{aligned}
\pi Q(2)= & \sum_{(i, u),(j, v)} g_{i} E_{0}^{(i, u)(j, v)} \otimes g_{j} E_{0}^{(j, v)} \\
= & \sum_{i, j, v}\left[g_{i} E_{v}^{i j} \otimes g_{j} E_{0}^{(j, v)}\right. \\
& \left.-\sum_{\substack{u \in \operatorname{Prev}(v) \\
k \neq i}} g_{i} \Gamma_{u}^{k i} E^{(i, u),(j, v)} \otimes g_{j} E_{0}^{(j, v)}\right] \\
= & \sum_{i, j, v} g_{i} \Gamma_{v}^{i j} \otimes g_{j} E_{0}^{(j, v)}-\pi \widehat{Q}(2) .
\end{aligned}
$$

For $n \geq 2$, substitution of $E_{t_{1}}^{k l_{1}}$ by the sum of two terms $\sum_{u \in \operatorname{Prev}\left(t_{1}\right)} E_{0}^{(k, u),\left(l_{1}, t_{1}\right)}$ and $\sum_{j \neq k, u \in \operatorname{Prev}\left(t_{1}\right)} \Gamma_{u}^{j k} E^{(k, u),\left(l_{1}, t_{1}\right)}$, one obtains:

$$
\begin{aligned}
\pi \widehat{Q}(n)= & \sum_{r \in \mathcal{S}(n)} g_{l_{1}} \sum_{k \notin \operatorname{List}^{1}(r)} \Gamma_{t_{1}}^{k l_{1}} E^{\left(l_{1}, t_{1}\right)\left(l_{2}, t_{2}\right)} \\
= & \sum_{\substack{\alpha \in \mathcal{S}(n) \\
k \notin \operatorname{List}^{1}(r)}}^{n-1} g_{\alpha} E^{\left(l_{\alpha}, t_{\alpha}\right),\left(l_{\alpha+1}, t_{\alpha+1}\right)} \otimes g_{l_{n}} E_{0}^{\left(l_{n}, t_{n}\right)} \\
& \otimes_{t_{1}}^{k-1} g_{\alpha=2} g_{\alpha} E^{\left(l_{\alpha}, t_{\alpha}\right),\left(l_{\alpha+1}, t_{\alpha+1}\right)} \otimes g_{l_{1}} E^{\left(l_{1}, t_{1}\right)\left(l_{2}, t_{2}\right)} \\
= & \pi \widehat{Q}(n+1)+\pi Q(n+1),
\end{aligned}
$$

where $\operatorname{List}(r)=\left\{\left(l_{1}, t_{1}\right), \cdots,\left(l_{n}, t_{n}\right)\right\}$. Equations (13) and (14) yield that

$$
\pi Q=\sum_{i} g_{i} F^{i}+\sum_{\substack{i, j \\ v \in \mathcal{T} \backslash \text { First }}} g_{i} \Gamma_{v}^{i j} \otimes g_{j} E_{0}^{(j, v)} .
$$

Using equations (7) and (6) to evaluate the second component of the right-hand side of (15) we get:

$$
\begin{aligned}
& R=\sum_{i, j, v} g_{i} \Gamma_{v}^{i j} \otimes g_{j} E_{0}^{(j, v)} \\
& =\sum_{i, j, v} g_{i} \Gamma_{v}^{i j} \otimes g_{j}\left(E_{0}^{(j, v)}-\sigma\left(E_{0}^{\left(j, \mathcal{T}^{a}\right)}\right)\right) \\
& +\sum_{i, j, v} g_{i}\left(E_{v}^{i j}-\sigma\left(E_{v}^{i j}\right)\right)+\sum_{i, j, v} g_{j} \Gamma_{v}^{i j} \sigma\left(E_{0}^{(j, v)}\right) \\
& +\sum_{i, j, v} g_{i} \sigma\left(E_{v}^{i j}\right)-\sum_{i, j, v} g_{j} \Gamma_{v}^{i j} \\
& =\sum_{i} g_{i}\left(H_{i}-F_{i}+\sigma\left(F_{i}\right)\right)+\sum_{i, j, v} g_{j} \Gamma_{v}^{i j} \sigma\left(E_{0}^{(j, v)}\right) \\
& +\sum_{i, j, v} g_{i} \sigma\left(\sum_{u \in \operatorname{Prev}(v)} E_{0}^{(i, u),(j, v)}\right. \\
& \left.+\sum_{k \neq i} \sum_{u \in \operatorname{Prev}(v)} \Gamma_{u}^{k i} E^{(i, u),(j, v)}\right)-\sum_{i, j, v} g_{j} \Gamma_{v}^{i j} \\
& =\sum_{i} g_{i}\left(-F_{i}+\sigma\left(F_{i}\right)\right)-\sum_{i, j, v} g_{j} \Gamma_{v}^{i j} \\
& \times\left[I d-\sigma\left(E_{0}^{(j, v)}+\sum_{\substack{k \neq j \\
t}} E^{(j, v)(k, t)}\right)\right] \\
& +\sum_{\substack{i, j, v \\
u \in \operatorname{Prev}(v)}} g_{i} \sigma\left(E_{0}^{(i, u),(j, v)}\right) \otimes g_{j} \sigma\left(E^{(j, v)}\right) \\
& =\sum_{i} g_{i}\left(-F_{i}+\sigma\left(F_{i}\right)\right)+\pi \sigma(Q(2))+\pi \sigma(\widetilde{Q}(2)) .
\end{aligned}
$$

For $n \geq 2$, using Equations (8), (9) and the definition of $E^{\left(l_{n}, t_{n}\right), k}$, one obtains:

$$
\begin{aligned}
\pi \sigma(\widetilde{Q}(n))= & \sum_{r \in \mathcal{S}(n)} g_{1} E_{0}^{\left(l_{1}, t_{1}\right),\left(l_{2}, t_{2}\right)} \\
& \otimes_{\alpha=2}^{n-1} g_{\alpha} E^{\left(l_{\alpha}, t_{\alpha}\right),\left(l_{\alpha+1}, t_{\alpha+1}\right)} \\
& \otimes g_{l_{n}} \sigma\left(\sum_{k \notin \operatorname{List}^{1}(r)} \sum_{t} E^{\left(l_{n}, t_{n}\right)(k, t)}\right) \\
= & \pi \sigma(Q(n+1))+\pi \sigma(\widetilde{Q}(n+1)) .
\end{aligned}
$$

where $\operatorname{List}(r)=\left\{\left(l_{1}, t_{1}\right), \cdots,\left(l_{n}, t_{n}\right)\right\}$. Finally Equations (15),(16) and (17) imply that:

$$
\pi Q=\pi \sigma(Q) .
$$

This completes the proof.

\subsection{The General Result}

The proof of theorem (4.1) without any restriction on the list is in the Appendix. Define

$$
\text { Last }=\left\{t \in \mathcal{T} \mid E_{0}^{(j, t),(i, u)}=E^{(j, t),(i, u)}=0, \forall j, i, u\right\}
$$

the set of all types can be present only at the last step.

In theorem (4.1), all the types in Last only appear in the hypothesis $H_{i} g_{i}=0$, with the term

$$
\Gamma_{\operatorname{Prev}\left(\mathcal{T}^{a}\right) \mathcal{T}^{a}}^{j i}\left(E_{0}^{\left(i, \mathcal{T}^{a}\right)}-\sigma\left(E_{0}^{\left(i, \mathcal{T}^{a}\right)}\right)\right) .
$$

Hence, one may consider a more general model (with more possibility of matrices): for a type $u$ in Last, $\{u\}=\mathcal{T}^{a}$, one can have several matrices $E_{0}^{(i, u)}$ depending on synchronization $r: E_{0}^{(i, u, r)}$ for each possible synchronization $r$. The 
assumptions we now need are: if $E_{0}^{(i, u, r)} \neq 0$ (which means synchronization $r$ at its last step at automaton $i$ and with type $u$ ), then:

$$
\begin{aligned}
& \sigma\left(E_{0}^{(i, u, r)}\right)=\sigma\left(E^{i, u}\right)=I d, \\
& g_{i} E_{0}^{(i, u, r)}=g_{i} E_{0}^{(i, u)} .
\end{aligned}
$$

With the above modification, the network still has product form solution.

\section{EXAMPLES}

Let us now consider some examples to show the effectiveness of the approach.

\subsection{A finite buffer network with jump over blocking, exponential services, Poisson ar- rivals}

Consider a network of finite buffer queues. At queue $i$, the capacity is $K_{i}$, the arrival rate is $\lambda_{i}$, the service rate is $\mu_{i}$. A customer after completion of its service at queue $i$ arrives to queue $j$ with probability $p_{i j}$ or leaves the queue $i$ immediately with probability $d_{i}$. When a customer arrives at queue $i$ and finds $K_{i}$ customers present, it jumps over the queue as a customer completing service and it routes to the next queue with the same probability.

Remember that: $\mathcal{T}=\{a, d, f\}, \mathcal{T}^{1}=\{a, f\}$, and $\operatorname{Prev}\left(\mathcal{T}^{1}\right)=$ $\{d, f\}$. Matrices $F_{i}$ which describe the local transitions are:

$$
F_{i}=\lambda_{i} U p_{K_{i}}+\lambda_{i} d_{i} I_{K_{i}}+\mu_{i} d_{i} \text { Low }_{K_{i}} .
$$

To define the synchronizations we use the following matrices:

$$
\begin{aligned}
& E_{0}^{(i, d),\left(j, \mathcal{T}^{1}\right)}=E_{0}^{(i, d),(j, a)}=E_{0}^{(i, d),(j, f)}=\mu_{i} p_{i j} \text { Low }_{K_{i}}, \\
& E_{0}^{(i, f),\left(j, \mathcal{T}^{1}\right)}=E_{0}^{(i, f),(j, a)}=E_{0}^{(i, f),(j, f)}=\lambda_{i} p_{i j} I_{K_{i}}, \\
& E^{(i, f),\left(j, \mathcal{T}^{1}\right)}=E^{(i, f),(j, f)}=E^{(i, f),(j, a)}=p_{i j} I_{K_{i}}, \\
& E_{0}^{i, a}=U p_{K_{i}}, \quad E_{0}^{i, f}=d_{i} I_{K_{i}},
\end{aligned}
$$

and all the other matrices are null. Matrix $E^{j, \mathcal{T}^{1}}$ is given by:

$$
E^{j, \mathcal{T}^{1}}=U p_{K_{i}}+d_{i} I_{K_{i}}+\sum_{j} p_{j i} I_{K_{i}}=U p_{K_{i}}+I_{K_{i}} .
$$
of:

We can rewrite Assumption (11) as $g_{i}$ being an eigenvector

$$
\begin{aligned}
E_{\{d, f\}\{a, f\}}^{i j}= & \mu_{i} p_{i j} \operatorname{Low}_{K_{i}}+\lambda_{i} p_{i j} I_{K_{i}} \\
& +\sum_{k} \Gamma_{\{d, f\}\{a, f\}}^{k, i} p_{i j} I_{K_{i}} .
\end{aligned}
$$

After substitution in the system, one obtains that $g_{i}$ has a geometric distribution with rate $\rho_{i}$ such that:

$$
\rho_{i}=\frac{\lambda_{i}+\sum_{j} \Gamma_{\{d, f\}\{a, f\}}^{j i}}{\mu_{i}},
$$

where $\Gamma_{\{d, f\}\{a, f\}}^{j i}=\mu_{j} \rho_{j} p_{j i}$. This is the typical flow equation in such a network of queues.

\subsection{Network with jump over blocking with a state dependent service rate}

Consider a network similar to the network in section 5.1, except that the service rate in queue $i$ is $\mu_{i}(n)$ when $n$ customers are present. Define matrix $D^{i}$ of size $K_{i}$ as:

$$
D^{i}=\left(\begin{array}{cccc}
0 & \cdots & \cdots & 0 \\
\mu_{i}(1) & 0 & \cdots & 0 \\
\cdots & \cdots & \cdots & \cdots \\
0 & \cdots & \mu_{i}\left(K_{i}\right) & 0
\end{array}\right) .
$$

One can deduce that $g_{i}$ is an eigenvector of $E_{\{d, f\}\{a, f\}}^{i j}$ :

$$
\frac{g_{i}(n) \mu_{i}(n) p_{i j}}{g_{i}(n-1)}=\Gamma_{\{d, f\}\{a, f\}}^{i j} .
$$

Solving the system after substitution, one can find that $g_{i}$ is given by:

$$
g_{i}(n)=g_{i}(0) \prod_{i=1}^{n} \frac{\Gamma^{i}}{\mu_{i}(n)},
$$

where $\Gamma_{i}$ satisfies the flow equations: $\Gamma_{i}=\lambda_{i}+\sum_{j} \Gamma_{j} p_{j i}$ and $\Gamma_{i} p_{i j}=\Gamma_{\{d, f\}\{a, f\}}^{i j}$.

\subsection{Network with negative signals}

Consider an open network of $N$ queue with exponential services and Poisson arrivals from the outside. The service rate in queue $i$ is $\mu_{i}$. Customers arrive to queue $i$ from the outside with rate $\lambda_{i}$. A customer departing from queue $i$ arrives at queue $j$ as a customer with probability $p_{i j}$, as a signal with probability $p_{i j^{-}}$, or leaves the network with probability $d_{i}$. A signal arrives to queue $i$ from the outside with rate $\lambda_{i}^{-}$. A signal arrives to queue $i$ will trigger a customer from queue $i$ to queue $j$, if there is, as a customer with probability $p_{i^{-} j}$, as a signal with probability $p_{i^{-} j^{-}}$. Therefore the first signal emitted from the queue can have an instantaneous effect on several queues because of the transformation of customers into signals. This is much more general than the Gnetworks with triggers where only one customer can be triggered. Remember that:

$$
\begin{array}{r}
\mathcal{T}=\{a, d, s\}, \quad \mathcal{T}^{1}=\{a\}, \quad \mathcal{T}^{2}=\{s\}, \\
\operatorname{Prev}\left(\mathcal{T}^{1}\right)=\operatorname{Prev}\left(\mathcal{T}^{2}\right)=\{d, s\} .
\end{array}
$$

Matrices $F_{i}$ are used to describe the local transitions:

$$
F_{i}=\lambda_{i} U p+\mu_{i} d_{i} L o w,
$$

while we model the synchronizations with the following matrices:

$$
\begin{aligned}
& E_{0}^{(i, d),(j, a)}=\mu_{i} p_{i j} \text { Low, } E_{0}^{(i, d),(j, s)}=\mu_{i} p_{i j^{-}} \text {Low, } \\
& E_{0}^{(i, s),(j, a)}=\lambda_{i}^{-} p_{i-j} \text { Low }, E_{0}^{(i, s),(j, s)}=\lambda_{i}^{-} p_{i^{-} j^{-}} \text {Low }, \\
& E^{(i, s),(j, a)}=p_{i-j} \text { Low, } E^{(i, s),(j, s)}=p_{i^{-} j^{-}} \text {Low, } \\
& E_{0}^{i, a}=U p, E_{0}^{i, s}=I^{0} .
\end{aligned}
$$

Furthermore $E^{j, a}=U p$ and $E^{j, s}=I^{0}+$ Low . One can rewrite Assumption (11) as $g_{i}$ being an eigenvector of both matrices:

$$
\begin{aligned}
E_{\{d, s\}\{a\}}^{i j}= & \left(\mu_{i} p_{i j}+\lambda_{i}^{-} p_{i^{-} j}\right) \text { Low } \\
& +\sum_{k} \Gamma_{\{d, s\}\{s\}}^{k, i} p_{i^{-} j} \text { Low, } \\
E_{\{d, s\}\{s\}}^{i j}= & \left(\mu_{i} p_{i j^{-}}+\lambda_{i}^{-} p_{i^{-} j^{-}}\right) \text {Low } \\
& +\sum_{k} \Gamma_{\{d, s\}\{s\}}^{k, i} p_{i^{-} j^{-}} \text {Low. }
\end{aligned}
$$


After substitution in the system, one obtains that $g_{i}$ has a geometric distribution with rate $\rho_{i}$ such that:

$$
\rho_{i}=\frac{\lambda_{i}+\sum_{j} \Gamma_{\{d, s\}\{a\}}^{j i}}{\mu_{i}+\lambda_{i}^{-}+\sum_{j} \Gamma_{\{d, s\}\{s\}}^{j i}},
$$

where

$$
\begin{aligned}
& \Gamma_{\{d, s\}\{a\}}^{j i}=\mu_{j} \rho_{j} p_{j i}+p_{j-i} \rho_{j}\left(\lambda_{j}^{-}+\sum_{k} \Gamma_{\{d, s\}\{s\}}^{k j}\right), \\
& \Gamma_{\{d, s\}\{s\}}^{j i}=\mu_{j} \rho_{j} p_{j i^{-}}+p_{j^{-} i^{-}} \rho_{j}\left(\lambda_{j}^{-}+\sum_{k} \Gamma_{\{d, s\}\{s\}}^{k j}\right) .
\end{aligned}
$$

REMARK 5.1. This is a generalization of the example introduced in [12]. The network consists of 3 queues. Customers arrive to queue $i$ from the outside with rate $\lambda_{i}$. The service rate at queue $i$ is $\mu_{i}$. The routing matrix is as follow: a customer completing service at queue 1 arrives to queue 2 as a signal, this signal will delete a customer at queue 2 and then moves to queue 3 as a signal. The signal will delete a customer at queue 3 and moves to queue 2 as a signal. This will repeat until queue 2 or queue 3 will be empty. In this case, the routing matrix is given by:

$$
p_{12^{-}}=p_{2^{-} 3^{-}}=p_{3^{-2^{-}}}=d_{2}=d_{3}=1 \text {. }
$$

Hence, $g_{i}$ has a geometric distribution with rate $\rho_{i}$

$$
\rho_{1}=\frac{\lambda_{1}}{\mu_{1}}, \quad \rho_{2}=\frac{\lambda_{2}}{\mu_{2}+\Gamma^{12}+\Gamma^{32}}, \quad \rho_{3}=\frac{\lambda_{3}}{\mu_{3}+\Gamma^{23}},
$$

where

$$
\Gamma^{12}=\mu_{1} \rho_{1}, \quad \Gamma^{32}=\rho_{3} \Gamma^{23}, \quad \Gamma^{23}=\rho_{2}\left(\Gamma^{12}+\Gamma^{32}\right) .
$$

And we obtain the usual flow equations.

\subsection{Network with positive signals}

In [2], positive signals were introduced. A positive signal will add a customer to queue where it arrives. The matrix corresponding is $U p$. If we want to use Theorem 4.1, the assumption that $g_{i}$ is an eigenvector of matrices $E$ leads us to this hypothesis that: when the queue is empty, there will be a positive signals activated. Consider a network of $N$ queues. The service rate in queue $i$ is $\mu_{i}$. Customers arrive to queue $i$ from the outside with rate $\lambda_{i}$. A departure from queue $i$ arrives to queue $j$ as a customer with probability $p_{i j}$, as a positive signal with probability $p_{i j^{+}}$, or leaves the network with probability $d_{i}$. A positive signal arrives to queue $i$ from the outside with rate $\lambda_{i}^{+}$. A positive signal arrives to queue $i$ will add a customer to queue $i$ and leaves to queue $j$ as a customer with probability $p_{i+}$, as a positive signal with probability $p_{i+}{ }^{+}$. One has:

$$
\begin{array}{r}
\mathcal{T}=\left\{a, d, s^{+}, e\right\}, \quad \mathcal{T}^{1}=\{a\}, \quad \mathcal{T}^{2}=\left\{s^{+}\right\}, \\
\operatorname{Prev}\left(\mathcal{T}^{1}\right)=\operatorname{Prev}\left(\mathcal{T}^{2}\right)=\left\{d, s^{+}, e\right\},
\end{array}
$$

where " $s^{+}$" and " $e$ " stand for positive signal and "empty". Matrices $F_{i}$ which describe the local transitions are:

$$
F_{i}=\lambda_{i} U p+\mu_{i} d_{i} \text { Low }
$$

Synchronizations are described by:

$$
\begin{aligned}
& E_{0}^{(i, d),(j, a)}=\mu_{i} p_{i j} \text { Low, } E_{0}^{(i, d),\left(j, s^{+}\right)}=\mu_{i} p_{i j} \text { Low, } \\
& E_{0}^{\left(i, s^{+}\right),(j, a)}=\lambda_{i}^{+} p_{i^{+}{ }_{j}} U p, \\
& E_{0}^{\left(i, s^{+}\right),\left(j, s^{+}\right)}=\lambda_{i}^{+} p_{i+}{ }_{j} U p, \\
& E_{0}^{(i, e),(j, a)}=C_{a r}^{i} I^{0} p_{i^{+} j}, E_{0}^{(i, e),\left(j, s^{+}\right)}=C_{a r}^{i} I^{0} p_{i^{+} j^{+}}, \\
& E^{\left(i, s^{+}\right),(j, a)}=p_{i^{+}{ }_{j}} U p, E^{\left(i, s^{+}\right),\left(j, s^{+}\right)}=p_{i^{+}{ }^{+}} U p, \\
& E_{0}^{i, a}=U p,
\end{aligned}
$$

where $C_{a r}^{i}$ is the active rate when the queue $i$ is empty. Matrices $E^{j, \mathcal{T}^{a}}$ are given by: $E^{j, a}=E^{j, s^{+}}=U p$. Now consider Assumption (11). We can restate it in terms of eigenvector: $g_{i}$ is an eigenvector of both matrices:

$$
\begin{aligned}
E_{\left\{d, s^{+}, e\right\}\{a\}}^{i j}= & \mu_{i} p_{i j} \text { Low }+\lambda_{i}^{+} p_{i^{+}{ }_{j}} U p \\
& +C_{a r}^{i} I^{0} p_{i^{+} j} \\
& +\sum_{k} \Gamma_{\left\{d, s^{+}, e\right\}\left\{s^{+}\right\}}^{k i} p_{i^{+} j} U p, \\
E_{\left\{d, s^{+} e\right\}\left\{s^{+}\right\}}^{i j}= & \mu_{i} p_{i j^{+}} \text {Low }+\lambda_{i}^{+} p_{i^{+} j^{+}} U p \\
& +C_{a r}^{i} I^{0} p_{i^{+} j^{+}} \\
& +\sum_{k} \Gamma_{\left\{d, s^{+}, e\right\}\left\{s^{+}\right\}}^{k i} p_{i^{+} j^{+}} U p .
\end{aligned}
$$

After substitution one obtains that $g_{i}$ has a geometric distribution with rate $\rho_{i}$ :

$$
\rho_{i}=\frac{\lambda_{i}+\sum_{j} \Gamma_{\left\{d, s^{+}, e\right\}\{a\}}^{j i}+\lambda_{i}^{+}+\sum_{j} \Gamma_{\left\{d, s^{+}, e\right\}\left\{s^{+}\right\}}^{j i}}{\mu_{i}},
$$

where

$$
\begin{aligned}
& \Gamma_{\left\{d, s^{+}, e\right\}\{a\}}^{j i}=\mu_{j} \rho_{j} p_{j i}+p_{j+i} C_{a r}^{j}, \\
& \Gamma_{\left\{d, s^{+}, e\right\}\left\{s^{+}\right\}}^{j i}=\mu_{j} \rho_{j} p_{j i}++p_{j+i^{+}} C_{a r}^{j},
\end{aligned}
$$

and $C_{a r}^{i}=\rho_{i}^{-1}\left(\lambda_{i}^{+}+\sum_{k} \Gamma_{\left\{d, s^{+}, e\right\}\left\{s^{+}\right\}}^{k i}\right)$. Again this is equivalent to a flow equation.

\subsection{Network with signals and jump over block- ing}

We now consider networks with negative signals, positive signals and "jump over blocking". Sets of type $\mathcal{T}, \mathcal{T}^{a}$ and Prev are defined by:

$$
\begin{aligned}
& \mathcal{T}=\left\{a, d, f, s, e, s^{+}, f^{+}\right\}, \\
& \mathcal{T}^{1}=\{a, f\}, \quad \mathcal{T}^{2}=\{s\}, \quad \mathcal{T}^{3}=\left\{s^{+}, f^{+}\right\}, \\
& \operatorname{Prev}\left(\mathcal{T}^{1}\right)=\operatorname{Prev}\left(\mathcal{T}^{2}\right)=\operatorname{Prev}\left(\mathcal{T}^{3}\right)=\mathcal{T} \backslash\{a\},
\end{aligned}
$$

where "f" stands for "full", $f$ and $f^{+}$are the "full state" of a queue corresponding to an arrival of a "normal customer" and a "positive signal", respectively.

The distribution $g_{i}$ has a geometric distribution with rate $\rho_{i}$ which is not dependent of the buffer size. When the buffer is infinite we still have the same value for $\rho_{i}$. The result is the combination of the ones obtained in the two previous sections: networks with negative signals and positive signals.

\subsection{Domino synchronization}

The SAN with Domino synchronization introduced in [9] is a special case of the synchronization multiple steps. The set of type $\mathcal{T}$ contain 3 elements: $\mathcal{T}=\{1,2,3\}$. The type 1 is associated with the first step, which is the Master, type 2 
with the second step (the Slave) and type 3 for the last step denoted as the Relay. Domino synchronization were defined in [9] with matrices $D, E$ and $T$ to represent respectively the effect of the synchronization on the Master, the Slave and the Relay. We can easily translate into the notations used here to model multiple step synchronizations:

$$
\begin{aligned}
& D^{r}-\sigma\left(D^{r}\right)=E_{0}^{(\text {master }, 1)(\text { slave }, 2)}, \\
& E_{1}^{r}=E^{(\text {slave }, 2),(\text { relay }, 3)}, \quad E_{2}^{r}=E_{0}^{(\text {slave }, 2)}, \\
& T^{r}=E_{0}^{(\text {relay }, 3)} .
\end{aligned}
$$

Sets $\mathcal{T}^{a}$ and Prev are given by:

$$
\mathcal{T}^{1}=\{2\}, \mathcal{T}^{2}=\{3\}, \operatorname{Prev}\left(\mathcal{T}^{1}\right)=\{1\}, \operatorname{Prev}\left(\mathcal{T}^{2}\right)=\{2\} .
$$

And matrices $E_{P \operatorname{rev}\left(\mathcal{T}^{a}\right) \mathcal{T}^{a}}^{i j}$ are obtained easily:

$$
\begin{aligned}
& E_{\{1\}\{2\}}^{\text {master,slave }}=D^{r}-\sigma\left(D^{r}\right), \\
& E_{\{2\}\{3\}}^{\text {slave,relay }}=\sum_{r^{\prime} \mid \text { slave }\left(r^{\prime}\right)=\text { slave }} \Gamma^{r^{\prime}} E_{1}^{r},
\end{aligned}
$$

and one has $\Gamma^{r}=\Gamma_{\{1\}\{2\}}^{\text {master,slave }}, \Omega^{r}=\frac{E_{\{2\}\{3\}}^{\text {slave, relay }}}{\sum_{r^{\prime} \mid \text { slave }\left(r^{\prime}\right)=\text { slaver }} \Gamma^{r^{\prime}}}$. Then, the domino synchronization is just one simple case of a synchronization with multiple steps.

\section{CONCLUDING REMARKS}

As SANs are very close to Stochastic Process Algebra (SPA), many results proved on SAN apply also on SPA We want to emphasis that some of the examples presented in section 5 may clearly be proved by Reversed Compound Agent Theorem (RCAT) Theorem [15] which is based on the Performance Evaluation Process Algebra (PEPA) SPA even if it is applied to Gnetworks of queues. But our objective here is to present a new type of proof which is purely algebraic while most of the results on SPA look for a probabilistic interpretation of the sufficient assumptions for product form. The theorem we prove here allows to generalize many results on queues with customers and signals. But the post important result is a simple algebraic proof based on tensor. As the tensor representation is not limited to SAN (see for instance $[6,16]$ ) we hope that this approach will lead to new research activities on the link between tensor representation and closed form solutions. Another approach is to find tensor representation for Zero Automatic queues [3].

This project is partially supported by a French ANR project (SETIN CheckBound).

\section{REFERENCES}

[1] R. Boucherie, "A Characterization of independence for competing Markov chains with applications to stochastic Petri nets", IEEE Trans. Software Eng., V20, N7, pp 536-544, 1994.

[2] X. Chao,M. Miyazawa and M. Pinedo. " Queueing Networks. Customers, Signals and Product Form Solutions", John Wiley \& Sons, Chichester, 1999.

[3] T-H Dao-Thi and J. Mairesse, "Zero-Automatic Networks", Discrete Event Dynamic Systems, V18, N4, 2008, pp 499-536.

[4] T-H Dao-Thi and J. M. Fourneau, "Stochastic Automata Networks with Master/Slave Synchronization: product form and tensor", ASMTA
2009, Madrid, Springer Verlag, LNCS 5513, pp 279-293.

[5] N.M. van Dijk, "On Jackson's product form with 'jump-over' blocking", 1988, Serie Research Memoranda VU University Amsterdam, Faculty of Economics, Business Administration and Econometrics, N0004.

[6] S. Donnatelli, "Superposed stochastic automata : a class of stochastic Petri nets with parallel solution and distributed state space", Performance Evaluation, V18, 1993, pp 21-36.

[7] P. Fernandes, B. Plateau, W.J. Stewart, "Efficient Descriptor-Vector Multiplications in Stochastic Automata Networks", JACM, 1998, pp 381-414.

[8] J.M. Fourneau, "Discrete Time Markov chains competing over resources: product form steady-state distribution", IEEE QEST 2008, pp 147-156.

[9] J.-M. Fourneau, "Product Form Steady-State Distribution for Stochastic Automata Networks with Domino Synchronizations", EPEW 2008, pp 110-124, Springer Verlag, LNCS 5261.

[10] J.M. Fourneau, B. Plateau, W. Stewart, "Product form for Stochastic Automata Networks", Proc. of ValueTools 2007, Nantes, France, 2007.

[11] J.M. Fourneau, B. Plateau, and W. Stewart, "An Algebraic Condition for Product Form in Stochastic Automata Networks without Synchronizations", Performance Evaluation, V65, N11-12, pp 854-868, 2008.

[12] J.M. Fourneau, F. Quessette. "Computing the steady-state distribution of G-networks with synchronized partial flushing", ISCIS 2006, Istanbul, Springer Verlag, LNCS 4263.

[13] E. Gelenbe and J.M. Fourneau, "G-networks with resets", Performance Evaluation, Vol. 49, No 1/4, pp 179-191, 2002 .

[14] E. Gelenbe, "Product form queueing networks with negative and positive customers", Journal of Applied Probability, V 28, pp 656-663, 1991.

[15] P. G. Harrison, "Compositional reversed markov processes with applications to G-networks", Performance Evaluation, vol. 57, pp. 379-408, July 2004.

[16] L. Kloul, J. Hillston,"An efficient Kronecker representation for PEPA models", PAPM 2001, L. de Alfaro, S. Gilmore. (eds.), PAPM-PROBMIV, vol. 2165 of LNCS, Springer, 2001.

[17] C.F. van Loan,"The Ubiquitous Kronecker Product", Journal of Computational and Applied Mathematics, pp. 85-100, vol. 123, 2000.

[18] B. Plateau, "On the Stochastic Structure of Parallelism and Synchronization Models for Distributed Algorithms", Proc. ACM Sigmetrics Conference on Measurement and Modeling of Computer Systems, Austin, Texas, August 1985.

[19] A. Lazar, T. Robertazzi, "Markovian Petri Net Protocols with Product Form Solution", Performance Evaluation, 1991, V12, pp 66-77. 


\section{APPENDIX}

\section{Proof of the theorem 4.1}

As mentioned before, we will prove that $\pi Q=\pi \sigma(Q)$. The idea is similar to the proof in section 4.2. Remember that

$$
\mathcal{S}(n)=\{r \in \mathcal{S}|| \operatorname{List}(r) \mid=n\}
$$

the set of all synchronizations with length $n$. Matrix $Q(n)$ is still given by

$$
Q(n)=\sum_{r \in \mathcal{S}(n)} M_{1}^{r} \otimes \cdots \otimes M_{N}^{r}
$$

however $\widehat{Q}(n)$ and $\widetilde{Q}(n)$ will be defined in a slightly different way. In the following definitions, for synchronization $r$ in $\mathcal{S}(n), \operatorname{List}(r)=\left\{\left(l_{1}, t_{1}\right), \cdots,\left(l_{n}, t_{n}\right)\right\}$. Now let us define:

$$
\begin{aligned}
& \widehat{Q}(n)=\sum_{r \in \mathcal{S}(n)} M_{1}^{r} \otimes \cdots \otimes \widehat{M}_{l_{1}}^{r} \cdots \otimes M_{N}^{r}, \\
& \widetilde{Q}(n)=\sum_{r \in \mathcal{S}(n)} M_{1}^{r} \otimes \cdots \otimes \widetilde{M}_{l_{n}}^{r} \cdots \otimes M_{N}^{r} .
\end{aligned}
$$

where

$$
\begin{aligned}
\widehat{M}_{l_{1}}^{r} & =\sum_{k \neq l_{1}} \sum_{t_{1} \in \mathcal{T}^{a}} \Gamma_{\operatorname{Prev}\left(\mathcal{T}^{a}\right) \mathcal{T}^{a}}^{k l_{1}} E^{\left(l_{1}, t_{1}\right),\left(l_{2}, t_{2}\right)} \frac{M_{l_{1}}^{r}}{M_{l_{1}}^{r}(1)}, \\
\widetilde{M}_{l_{n}}^{r} & =\frac{M_{l_{n}}^{r}}{M_{l_{n}}^{r}\left(\left|\operatorname{Order}\left(l_{n}, r\right)\right|\right)} \sum_{k \neq l_{n}, a \leq T} E^{\left(l_{n}, t_{n}\right),\left(k, \mathcal{T}^{a}\right)} .
\end{aligned}
$$

Equations (11) and $g_{i} E_{\operatorname{Prev}\left(\mathcal{T}^{a}\right) \mathcal{T}^{a}}^{i j}=\Gamma_{\operatorname{Prev}\left(\mathcal{T}^{a}\right) \mathcal{T}^{a}}^{i j} g_{i}$ imply that:

$$
\begin{aligned}
\pi Q(2)= & \sum_{(i, u),(j, v)} g_{i} E_{0}^{(i, u)(j, v)} \otimes g_{j} E_{0}^{(j, v)} \\
= & \sum_{i, j, \mathcal{T}^{a}}\left[g_{i} E_{\operatorname{Prev}\left(\mathcal{T}^{a}\right) \mathcal{T}^{a}}^{i j} \otimes g_{j} E_{0}^{\left(j, \mathcal{T}^{a}\right)}\right. \\
& \left.-\sum_{\substack{u \in \operatorname{Prev}\left(\mathcal{T}^{a}\right) \\
u \in \mathcal{T}^{b}, k \neq i}} g_{i} \Gamma_{\operatorname{Prev}\left(\mathcal{T}^{b}\right) \mathcal{T}^{b}}^{k i} E^{(i, u),\left(j, \mathcal{T}^{a}\right)} \otimes g_{j} E_{0}^{\left(j, \mathcal{T}^{a}\right)}\right] \\
= & \sum_{i, j, \mathcal{T}^{a}} g_{i} \Gamma_{\operatorname{Prev}\left(\mathcal{T}^{a}\right), \mathcal{T}^{a}}^{i j} \otimes g_{j} E_{0}^{\left(j, \mathcal{T}^{a}\right)}-\pi \widehat{Q}(2) .
\end{aligned}
$$

For $n \geq 2$, it is now more complicated to prove equation (14),

$$
\begin{aligned}
& \pi \widehat{Q}(n)=\sum_{|L i s t(r)|=n} \otimes_{i \neq l_{1}} g_{i} M_{i}^{r} \\
& \otimes g_{l_{1}} \sum_{\substack{k \neq l_{1} \\
t_{1} \in \mathcal{T}^{a}}} \Gamma_{\operatorname{Prev}\left(\mathcal{T}^{a}\right) \mathcal{T}^{a}}^{k l_{1}} E^{\left(l_{1}, t_{1}\right)\left(l_{2}, t_{2}\right)} \\
& =\sum_{\substack{\left|L_{i s t}(r)\right|=n \\
k \neq l_{1}, t_{1} \in \mathcal{T}^{a}}} \otimes M_{\substack{i \neq k \\
i \neq l_{1}}} g_{i} M_{i}^{r} \\
& \otimes g_{k} E_{\operatorname{Prev}\left(\mathcal{T}^{a}\right) \mathcal{T}^{a}}^{k l_{1}} M_{k}^{r} \\
& \otimes g_{l_{1}} E^{\left(l_{1}, t_{1}\right)\left(l_{2}, t_{2}\right)} M_{l_{1}}^{r}(2) \cdots M_{l_{1}}^{r}(m) \\
& =\pi \widehat{Q}(n+1)+\pi Q(n+1),
\end{aligned}
$$

where $\operatorname{List}(r)=\left\{\left(l_{1}, t_{1}\right), \cdots,\left(l_{n}, t_{n}\right)\right\}$ and $m=\left|\operatorname{Order}\left(l_{1}, r\right)\right|$. Equations (18) and (19) yield that:

$$
\pi Q=\sum_{i} g_{i} F^{i}+\sum_{i, j, \mathcal{T}^{a}} g_{i} \Gamma_{\operatorname{Prev}\left(\mathcal{T}^{a}\right) \mathcal{T}^{a}}^{i j} \otimes g_{j} E_{0}^{\left(j, \mathcal{T}^{a}\right)} .
$$

Using equations (7) and (6) to evaluate the second component of the right-hand side of (20) we get:

$$
\begin{aligned}
& R=\sum_{i, j, \mathcal{T}^{a}} g_{i} \Gamma_{\operatorname{Prev}\left(\mathcal{T}^{a}\right) \mathcal{T}^{a}}^{i j} \otimes g_{j} E_{0}^{\left(j, \mathcal{T}^{a}\right)} \\
& =\sum_{i, j, \mathcal{T}^{a}} g_{i} \Gamma_{\operatorname{Prev}\left(\mathcal{T}^{a}\right) \mathcal{T}^{a}}^{i j} \otimes g_{j}\left(E_{0}^{\left(j, \mathcal{T}^{a}\right)}-\sigma\left(E_{0}^{\left(j, \mathcal{T}^{a}\right)}\right)\right) \\
& +\sum_{i, j, \mathcal{T}^{a}} g_{i}\left(E_{\operatorname{Prev}\left(\mathcal{T}^{a}\right) \mathcal{T}^{a}}^{i j}-\sigma\left(E_{\operatorname{Prev}\left(\mathcal{T}^{a}\right) \mathcal{T}^{a}}^{i j}\right)\right) \\
& +\sum_{i, j, \mathcal{T}^{a}} g_{j} \Gamma_{\operatorname{Prev}\left(\mathcal{T}^{a}\right) \mathcal{T}^{a}}^{i j} \sigma\left(E_{0}^{\left(j, \mathcal{T}^{a}\right)}\right) \\
& +\sum_{i, j, \mathcal{T}^{a}} g_{i} \sigma\left(E_{\operatorname{Prev}\left(\mathcal{T}^{a}\right) \mathcal{T}^{a}}^{i j}\right)-\sum_{i, j, \mathcal{T}^{a}} g_{j} \Gamma_{\operatorname{Prev}\left(\mathcal{T}^{a}\right) \mathcal{T}^{a}}^{i j} \\
& =\sum_{i} g_{i}\left(H_{i}-F_{i}+\sigma\left(F_{i}\right)\right)-\sum_{i, j, \mathcal{T}^{a}} g_{j} \Gamma_{\operatorname{Prev}\left(\mathcal{T}^{a}\right) \mathcal{T}^{a}}^{i j} \\
& +\sum_{i, j, \mathcal{T}^{a}} g_{j} \Gamma_{\operatorname{Prev}\left(\mathcal{T}^{a}\right) \mathcal{T}^{a}}^{i j} \sigma\left(E_{0}^{\left(j, \mathcal{T}^{a}\right)}\right) \\
& +\sum_{i, j, \mathcal{T}^{a}} g_{i} \sigma\left(\sum_{u \in \operatorname{Prev}\left(\mathcal{T}^{a}\right)} E_{0}^{(i, u),\left(j, \mathcal{T}^{a}\right)}\right. \\
& \left.+\sum_{k \neq i} \sum_{\substack{u \in \operatorname{Prev}\left(\mathcal{T}^{a}\right) \\
u \in \mathcal{T}^{b}}} \Gamma_{\operatorname{Prev}\left(\mathcal{T}^{b}\right) \mathcal{T}^{b}}^{k i} E^{(i, u),\left(j, \mathcal{T}^{a}\right)}\right) \\
& =\sum_{i} g_{i}\left(-F_{i}+\sigma\left(F_{i}\right)\right)-\sum_{i, j, \mathcal{T}^{a}} g_{j} \Gamma_{\operatorname{Prev}\left(\mathcal{T}^{a}\right) \mathcal{T}^{a}}^{i j} \\
& \times\left[I d-\sigma\left(E_{0}^{\left(j, \mathcal{T}^{a}\right)}+\sum_{\substack{k \neq j \\
v \in \mathcal{T}^{a}, b \leq T}} E^{(j, v)\left(k, \mathcal{T}^{b}\right)}\right)\right] \\
& +\sum_{\substack{i, j, \mathcal{T}^{a} \\
u \in \operatorname{Prev}\left(\mathcal{T}^{a}\right)}} g_{i} \sigma\left(E_{0}^{(i, u),\left(j, \mathcal{T}^{a}\right)}\right) \otimes g_{j} \sigma\left(E^{\left(j, \mathcal{T}^{a}\right)}\right) \\
& =\sum_{i} g_{i}\left(-F_{i}+\sigma\left(F_{i}\right)\right)+\pi \sigma(Q(2))+\pi \sigma(\widetilde{Q}(2)) .
\end{aligned}
$$

For $n \geq 2$, using Equations (8), (9) and the definition of $E^{\left(l_{n}, t_{n}\right), k}$, one obtains:

$$
\begin{aligned}
\pi \sigma(\widetilde{Q}(n))= & \sum_{|L i s t(r)|=n} \otimes_{i \neq l_{n}} g_{i} \sigma\left(M_{i}^{r}\right) \\
& \otimes g_{l_{n}} \sigma\left(M_{l_{n}}^{r}(1) \cdots M_{l_{n}}^{r}(m-1)\right. \\
& \left.\times \sum_{\substack{k \neq l_{n} \\
a \leq T}} E^{\left(l_{n}, t_{n}\right)\left(k, \mathcal{T}^{a}\right)}\right) \\
= & \sum_{\substack{|\operatorname{List}(r)|=n \\
k \neq l_{n}, a \leq T}} \otimes_{i \notin\left\{k, l_{n}\right\}} g_{i} \sigma\left(M_{i}^{r}\right) \otimes g_{l_{n}} \\
& \sigma\left(M_{l_{n}}^{r}(1) \cdots M_{l_{n}}^{r}(m-1) E^{\left(l_{n}, t_{n}\right)\left(k, \mathcal{T}^{a}\right)}\right) \\
& \otimes g_{k} \sigma\left(M_{k}^{r} E^{\left(l_{n}, t_{n}\right), k}\right) \\
= & \pi \sigma(Q(n+1))+\pi \sigma(\widetilde{Q}(n+1)) .
\end{aligned}
$$

where List $(r)=\left\{\left(l_{1}, t_{1}\right), \cdots,\left(l_{n}, t_{n}\right)\right\}$ and $m=\left|\operatorname{Order}\left(l_{n}, r\right)\right|$. Equations (20),(21) and (22) imply:

$$
\pi Q=\pi \sigma(Q) .
$$

And the proof is completed. 